\title{
A CONTINUOUS MODEL OF A STANDING HUMAN BODY IN VERTICAL VIBRATION
}

\author{
Qingwen Zhang ${ }^{1,2^{*}}-$ Yu Zhang ${ }^{1,2}-$ Tianjian $\mathrm{Ji}^{3}$
}

${ }^{1}$ Key Lab of Structures Dynamic Behavior and Control of China Ministry of Education, Harbin Institute of Technology, Harbin 150090, China;

${ }^{2}$ School of Civil Engineering, Harbin Institute of Technology, Harbin 150090, China

${ }^{3}$ School of Mechanical, Aerospace and Civil Engineering, The University of Manchester, UK.

\begin{tabular}{l} 
ARTICLE INFO \\
\hline Article history: \\
Received: 1.8 .2016$. \\
Received in revised form: 10.12 .2016$. \\
Accepted: 16.12 .2016$. \\
\hline Keywords: \\
Human body models \\
Human-Structure interaction \\
\hline DOI: http://doi.org/10.30765/er.39.2.2
\end{tabular}

\section{Introduction}

In order to help understand human perception of structural vibration, models of a human body need to be provided correctly. A standing human body is a continuum in which the mass and stiffness of the body are distributed unevenly throughout the height of the body. To represent the standing body, many models were developed to represent the whole body. There may be four typical types of body models that were developed in different ways and have respective advantages and disadvantages:

\begin{abstract}
:
This paper develops a continuous standing human body model in the vertical vibration based on an anthropomorphic model, two measured natural frequencies of a biomechanics model, and structural dynamics methods. The mass distribution of a standing body is formed using the mass distribution of fifteen body segments in the anthropomorphic model. The axial stiffness of the model is determined based on the best matching to the two natural frequencies of the biomechanics model which were obtained using shaking table tests. Four similar models are assessed using finite element parametric analysis. The best of the four models has seven uniform mass segments with two stiffnesses and the same fundamental natural frequency as that of the biomechanics model, but its second natural frequency is $10 \%$ higher. The mode shapes of the continuous model are presented to demonstrate the relative magnitude of vibration throughout the height of the body. Finally the modal mass and stiffness of the continuous model are evaluated, which are related to some simple discrete models.
\end{abstract}

1. Single degree-of-freedom (SDOF) models, two SDOF Models and two degrees-of-freedom (TDOF) models: These models captured from the study of body biomechanics of seating and standing subjects using a shaking table [1-2]. The format of these models was intuitively provided while the parameters of the models, such as damping coefficients, stiffnesses and masses, were identified based on the best fitting between the measured and predicted apparent masses [3-4]. These models captured the biomechanical or dynamic characteristics of a whole-body. The two SDOF

\footnotetext{
${ }^{*}$ Qingwen Zhang, Tel.: +8645186282080; fax: +8645186283097

E-mail address: zhangqw@hit.edu.cn
} 
model is shown in Fig. 1a, and the parameters of the model are shown in Table 1, including the mass and frequencies. When examining the apparent mass of a stationary body on a vibrating structure, these models are ideal for either theoretical or experimental investigation.

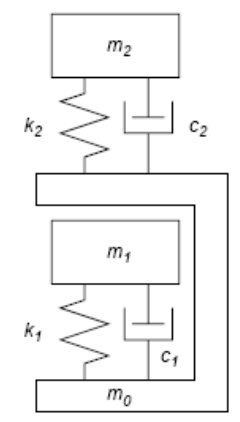

a) Biomechanics model (Model 2d)

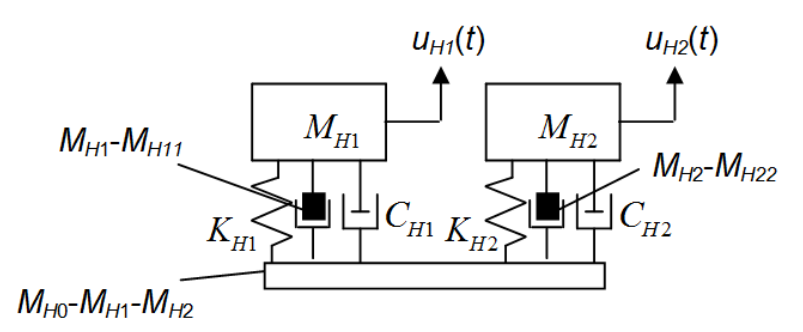

b) human-structure interaction model

Figure 1: Models of a standing body [4-6].

Table 1: Masses and natural frequencies of the body Models in Fig.1a [4]

\begin{tabular}{|c|c|}
\hline & Model 2d \\
\hline$m_{0}$ & 0.0909 \\
\hline$m_{1}$ & 0.254 \\
\hline$m_{2}$ & 0.655 \\
\hline Sum of the mass & 1.000 \\
\hline$f_{\mathrm{H} 1}(\mathrm{~Hz})$ & 5.88 \\
\hline$f_{\mathrm{H} 2}(\mathrm{~Hz})$ & 13.5 \\
\hline$\delta_{0}=f_{\mathrm{H} 2} / f_{\mathrm{H} 1}$ & 2.29 \\
\hline
\end{tabular}

2. Higher degrees of freedom models: When a human body is represented by three or more degrees of freedom models, the difficulties for determining the parameters of the models are unavoidable. If more degrees of freedom are considered, the representation of the body appears more reasonable, but it becomes even harder to define/give correct parameters of the body. Nigam and Malik [7] provided a 15 DOF spring mass system. This model was based on an anthropomorphic model of the average male body in a standing posture with the body modelled using ellipsoidal segments. The provision of the mass distribution might be reasonable but it would be extremely difficult to define accurately the stiffnesses of the fourteen springs linking the 15 masses.

3. Continuous body models: It is reasonable to represent a standing body as a continuous model because the body is a continuum. The difficulties for the continuous model are the provision of the mass and stiffness distributions along the height of the body. Ji [8] employed a continuous bar with two segments of different masses and stiffnesses (Fig. 3a) to simulate the vertical vibration of the body. The heights of the upper and lower parts of people were the same and the mass of the upper part was twice of that of the lower part. The mass distribution was simplified based on the data provided by Nigam and Malik [7]. The unknowns in the model were the stiffnesses of the lower and upper parts of the body. An assumption on the ratio of the two stiffnesses of the body was given and parametric studies were conducted. The continuous model allowed examining the vertical vibration along the height of a standing body but it was a pure theoretical study. The model might be more useful if experimental results could be used to determine the unknowns in the model.

4. Human-structure interaction models: These models were developed when a standing body was placed on the SDOF structure [5]. Interaction models that were developed based on a vibrating structure. The two SDOF models are shown in Fig. 1b. The parameters of the model were defined by exact mathematical expressions, giving a qualitatively correct model [9-10]. However, the parameters of the model shown in Fig. 1b cannot be derived without assumptions of the distributions of mass and stiffness throughout the height of a standing body and the mode shapes.

It can be seen that there are no links between the four types of model although they all represent a standing body and that these models complement to each other. This paper aims to represent a standing body as a continuous model by adopting the mass distribution developed by Bartz and Gianotti [11] and Nigam and Malik [7] using the two natural frequencies of a standing body in Table 1 [4]. This 
adoption would help to determine the unknown parameters in the continuous model, make the model more accurate, and show the vibration along the height of the standing body. In addition, relationships between the continuous and simple discrete models are discussed.

\section{A Continuous Standing Body Model}

\subsection{Assumptions}

To establish a human body model, it is important to present and clarify the basic assumptions involved in its development. The assumptions used in establishing the model and justification of the assumptions are given as follows:

- Local vibrations in a standing body are neglected. This model considers only global behavior which may be used to investigate the responses of a standing individual or a crowd and of the structure that supports the people. Thus the local vibrations of the human body, such as arm vibration or eyeball vibration, are insignificant for the purpose of the study and can be neglected.

- Each of the fifteen segments of a body has a uniform density. The standing body consists of 15 mass segments in this study, which was defined in the paper by Bartz and Gianotti [11] and Nigam and Malik [7]. The densities of the segments are different. However, it is assumed that each segment has a uniformly distributed density.

- The first two natural frequencies of a typical standing body (Table 1) adopted in this study are considered to be correct. The first two natural frequencies of the human body were obtained from shaking table tests [4]. They are considered to be correct and reliable. Thus they are directly used as a basis for identifying the two stiffness values in the continuous models of a standing body.

- The axial stiffness of a standing body is represented by constant upper and lower body stiffnesses. This assumption is likely to affect the accuracy of the model. However, only two known natural frequencies of a standing body are available, which restrict the model from having more unknown parameters.

Based on the above assumptions, the vertical vibration of the human body can be studied as the axial vibration of a column assembled from several uniform bars having different properties.

\subsection{Mass and stiffness distributions of the model}

Table 2 summarizes the mass distribution of the fifteen segments based on the papers of Bartz and Gianotti [11] and Nigam and Malik [7]. The second column in Table 2 describes the segments and the third and fourth columns show the mass and length of each segment. The left half of Fig. 2a shows a standing body where the values of segmental masses are indicated together with the lengths of the segments. Based on the second assumption, the mass density along the height is the ratio of the mass to the corresponding length of the segment. The right half of Fig. 2a shows the distribution of mass density along the height of the body and the combination of some segments. For example, the two arms and the upper torso are grouped as the arms connect to the upper torso. As only the vertical vibration of the body is to be studied, the two legs are merged into one in the model. Therefore, the fifteen segments are grouped into seven parts along the height of the body and each part has the same mass density. Figure $2 \mathrm{~b}$ shows the mass distribution of the continuous standing body model.

Incorporating the stiffness into the continuous model, four possible models that are summarized in the last four columns in Table 2 and described as follows:

Model 1: The upper nine segments are grouped into the upper part of the body, while the lower six segments are classified to the lower part. Each of the parts has the uniform mass distribution. Two different stiffnesses are assigned to the upper and lower parts respectively, as shown in Fig. 3a. It can be noted from the fifth column in Table 2 that the upper and lower parts have almost the same height. This model was originally developed by $\mathrm{Ji}$ [8] and was relatively simple. The reason to lump the fifteen segments into the upper and lower parts is that only two stiffness values can be assigned based on the fourth assumption and each part is given the same mass and stiffness.

Model 2: This model takes the mass distribution defined in Fig. $2 b$, i.e. the model has seven different mass density distributed along the height of the body. The two axial stiffnesses are assigned to the same heights as that in Model 1. The model appears more accurate than Model 1 as the mass distribution is more reasonable than that of Model 1.

Model 3: This model is almost the same as Model 2 except the assignment of the stiffness. It can be seen from the seventh column in Table 2 and Fig. $3 \mathrm{c}$ that 


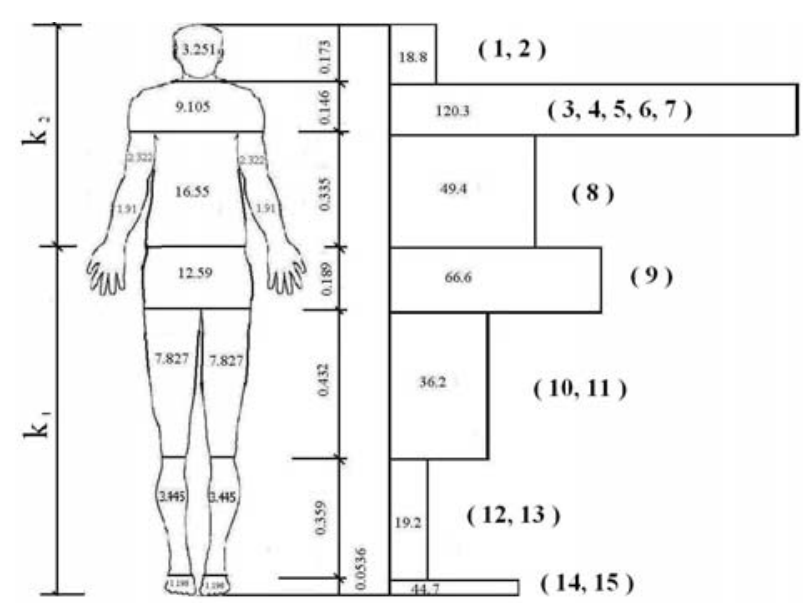

a) Anthropomorphic model and densities of the main parts of a body $(\mathrm{kg} / \mathrm{m})$

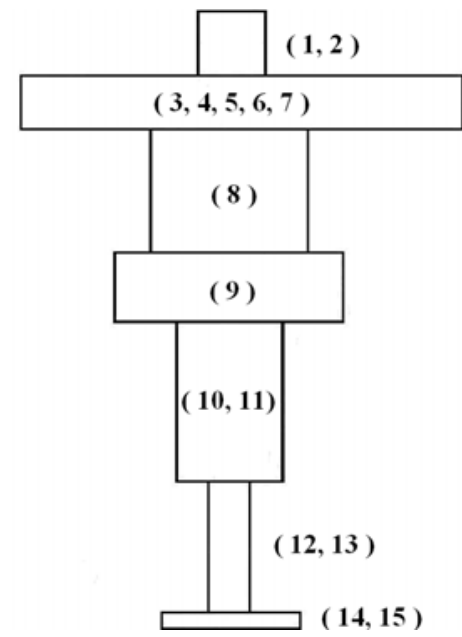

b) Continuous body model with known masses and unknown stiffness

Figure 2: Distribution of body mass.

Table 2. Mass distribution of 15 ellipsoidal segments [7,11]

\begin{tabular}{|c|c|c|c|c|c|c|c|}
\hline \multirow[t]{2}{*}{ No. } & \multirow{2}{*}{$\begin{array}{c}\text { Segment } \\
\text { designation }\end{array}$} & \multicolumn{2}{|c|}{$\begin{array}{c}\text { Anthropomorphic } \\
\text { Model }\end{array}$} & \multirow{2}{*}{$\begin{array}{c}\text { Model } 1 \\
M(\mathrm{~kg}) \\
L(\mathrm{~m})\end{array}$} & \multirow{2}{*}{$\begin{array}{c}\text { Model } 2 \\
M(\mathrm{~kg}) \\
L(\mathrm{~m})\end{array}$} & \multirow{2}{*}{$\begin{array}{c}\text { Model } 3 \\
M(\mathrm{~kg}) \\
L(\mathrm{~m})\end{array}$} & \multirow{2}{*}{$\begin{array}{c}\text { Model } 4 \\
M(\mathrm{~kg}) \\
L(\mathrm{~m})\end{array}$} \\
\hline & & $M_{(\mathrm{kg})}$ & $L(\mathrm{~m})$ & & & & \\
\hline 1 & Head pivot & 3.044 & 0.154 & \multirow{5}{*}{$\begin{array}{l}M_{1}^{1}= \\
49.96\end{array}$} & $M_{1}^{2}=3.251$ & $M_{1}^{3}=3.251$ & \\
\hline 2 & Neck pivot & 0.207 & 0.019 & & $L_{1}^{2}=0.173$ & $L_{1}^{3}=0.173$ & $M_{1}^{4}=$ \\
\hline 3 & Right upper arm & 2.322 & 0.291 & & \multirow{5}{*}{$\begin{aligned} M_{2}^{2} & =17.57 \\
L_{2}^{2} & =0.146\end{aligned}$} & \multirow{5}{*}{$\begin{array}{c}M_{2}^{3}=17.57 \\
L_{2}^{3}=0.146\end{array}$} & 37.37 \\
\hline 4 & Left upper arm & 2.322 & 0.291 & & & & \\
\hline 5 & Right lower arm & 1.910 & 0.378 & & & & $L_{1}^{4}=$ \\
\hline 6 & Left lower arm & 1.910 & 0.378 & \multirow{2}{*}{$\begin{array}{c}L_{1}^{1}= \\
0.842\end{array}$} & & & 0.653 \\
\hline 7 & Upper torso & 9.105 & 0.146 & & & & \\
\hline 8 & Centre torso & 16.55 & 0.334 & \multirow[t]{2}{*}{$k_{2}$} & $\begin{array}{c}M_{3}^{2}=16.55 \\
L_{3}^{2}=0.334\end{array}$ & $\begin{aligned} M_{3}^{3} & =16.55 \\
L_{3}^{3} & =0.334\end{aligned}$ & $k_{2}$ \\
\hline 9 & Lower torso & 12.59 & 0.189 & & $\begin{array}{c}M_{4}^{2}=12.59 \\
L_{4}^{2}=0.189\end{array}$ & $\begin{aligned} M_{4}^{3} & =12.59 \\
L_{4}^{3} & =0.189\end{aligned}$ & $M^{4}=$ \\
\hline 10 & Right upper leg & 7.827 & 0.432 & \multirow{6}{*}{$\begin{array}{l}M_{2}^{1}= \\
24.94\end{array}$} & $M_{5}^{2}=15.65$ & $M_{5}^{3}=15.65$ & 37.53 \\
\hline 11 & Left upper leg & 7.827 & 0.432 & & $L_{5}^{2}=0.432$ & $L_{5}^{3}=0.432$ & \\
\hline 12 & Right Lower leg & 3.445 & 0.359 & & $M_{6}^{2}=6.890$ & $M_{6}^{3}=6.890$ & $\begin{array}{l}L_{2}= \\
1.034\end{array}$ \\
\hline 13 & Left Lower leg & 3.445 & 0.359 & & $L_{6}^{2}=0.359$ & $L_{6}^{3}=0.359$ & \\
\hline 14 & Right foot & 1.198 & 0.054 & & $M_{7}^{2}=2.396$ & $M_{7}^{3}=2.396$ & $k_{1}$ \\
\hline \multirow[t]{2}{*}{15} & Left foot & 1.198 & 0.054 & & $L_{7}^{2}=0.054$ & $L_{7}^{3}=0.054$ & \\
\hline & Sum & 74.9 & 1.687 & $\begin{array}{l}M=74.9 \\
L=1.687\end{array}$ & $\begin{array}{l}M=74.9 \\
L=1.687\end{array}$ & $\begin{array}{l}M=74.9 \\
L=1.687\end{array}$ & $\begin{array}{l}M=74.9 \\
L=1.687\end{array}$ \\
\hline
\end{tabular}




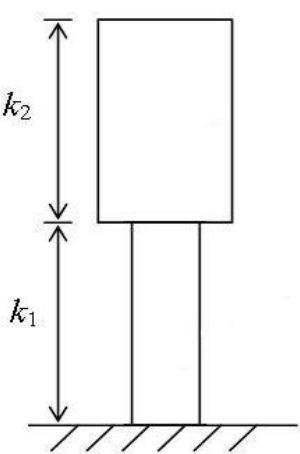

a) Model 1

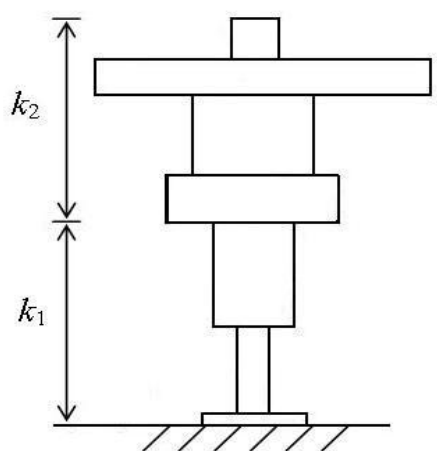

b) Model 2

Figure 3: Continuous standing body models.

stiffness $k_{1}$ is assigned to the four lower parts in this model while to the three lower parts in Model 2 . Interestingly, the ratio of the four lower parts to the height of the human body is 0.613 (approximately equal to the golden ratio 0.618). Model 4: This model is similar to Model 1 as the masses are distributed into only upper and lower parts. The difference is that the lower part includes the lower torso in this model while the upper part contains the lower torso in Model 1. Consequently, the stiffness distribution is altered too as shown in Fig.s $3 \mathrm{a}$ and $3 \mathrm{~d}$.

For the four models defined above, the mass distribution are known and the pattern of stiffness distribution is given, while the values of the two stiffnesses $k_{1}$ and $k_{2}$ are unknown and are to be determined in the next subsection.

\section{Identification of the Stiffness}

\subsection{The method of identification}

Parametric free vibration analysis is conducted to identify the two stiffnesses for the four models using the finite element method. For modelling the vertical vibration of the models, 338 bar elements (LINK 8) in ANSYS are used.

In the free vibration analysis, the stiffness of the models is normally the input, the same as the mass, and the natural frequency is the output. However, it is unlikely that the stiffness of the models can be determined directly from the two given natural frequencies. Thus, it would be helpful to understand the dynamic characteristics of the models before any parametric finite element analysis and the
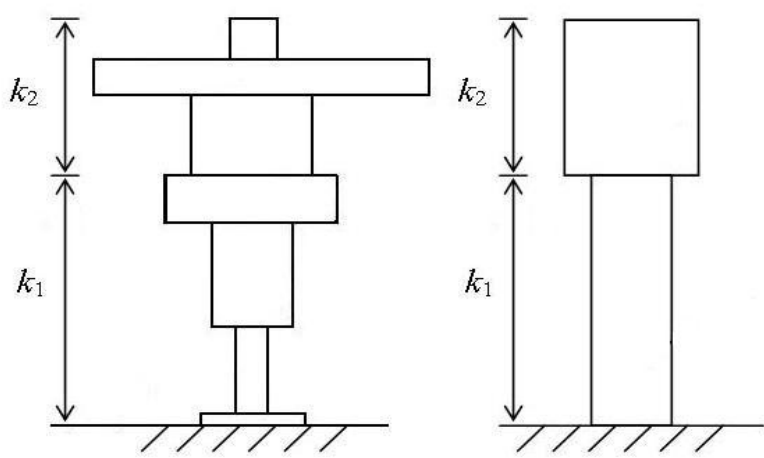

c) Model 3

d) Model 4

identification are conducted. The first two natural frequencies of the body models can be qualitatively expressed as:

$$
f_{\mathrm{i}}=\frac{1}{2 \pi} \sqrt{\frac{\int_{0}^{L} k(x)\left(\frac{\partial \phi_{\mathrm{i}}(x)}{\partial x}\right)^{2} \mathrm{~d} x}{\int_{0}^{L} m(x) \partial \phi_{1}(x)^{2} \mathrm{~d} x}} ; \quad \mathrm{i}=1,2
$$

Where $k(x)$ and $m(x)$ are the stiffness and mass distributions along the height of the body models; $\phi_{i}(x)$ is the shape function of the $i^{\text {th }}$ mode. If the true $k(x), m(x)$ and $\phi_{i}(x)$ can be provided, it will lead to the exact solution of the natural frequency. Due to the complexity of a human body, it is difficult for these functions to be determined.

The ratio of the second natural frequency to the first natural frequency is

$$
\frac{f_{2}}{f_{1}}=\sqrt{\frac{\int_{0}^{L} k(x)\left(\frac{\partial \phi_{2}(x)}{\partial x}\right)^{2} \mathrm{~d} x}{\int_{0}^{L} k(x)\left(\frac{\partial \phi_{1}(x)}{\partial x}\right)^{2} \mathrm{~d} x} \times \frac{\int_{0}^{L} m(x) \partial \phi_{1}(x)^{2} \mathrm{~d} x}{\int_{0}^{L} m(x) \partial \phi_{2}(x)^{2} \mathrm{~d} x}}
$$

For the study cases, the stiffness can be expressed as

$$
\begin{gathered}
k(x)=\left\{\begin{array}{l}
k_{1} \\
k_{2}
\end{array}=k_{1}\left\{\begin{array}{l}
1 \\
\frac{k_{2}}{k_{1}}
\end{array}=k_{1} S(x)\right.\right. \\
0<x \leq \beta L \quad \text { (lower part) } \\
\beta L<x \leq L \quad \text { (upper part) }
\end{gathered}
$$

where 


$$
S(x)= \begin{cases}1 & 0<x \leq \beta L \\ \frac{k_{2}}{k_{1}} & \beta L<x \leq L\end{cases}
$$

and $\beta$ is the ratio of the length of the lower part to the height of the model. Substituting the Equation 3 into the Equation 2 gives

$$
\frac{f_{2}}{f_{1}}=\sqrt{\frac{\int_{0}^{L} S(x)\left(\frac{\partial \phi_{2}(x)}{\partial x}\right)^{2} \mathrm{~d} x}{\int_{0}^{L} S(x)\left(\frac{\partial \phi_{1}(x)}{\partial x}\right)^{2} \mathrm{~d} x} \times \frac{\int_{0}^{L} m(x) \partial \phi_{1}(x)^{2} \mathrm{~d} x}{\int_{0}^{L} m(x) \partial \phi_{2}(x)^{2} \mathrm{~d} x}}
$$

It can be observed from the Equations 1, 4 and 5 that:

The equation 1 indicates that if $k(x)$ is scaled to $c^{2} k(x)$, the natural frequency $f_{\mathrm{i}}$ becomes $c f_{\mathrm{i}}$ where $\mathrm{c}$ is a constant.

The equations 4 and 5 indicate that the frequency ratio $f_{2} / f_{1}$ only relates to the stiffness ratio $k_{2} / k_{1}$ rather than the absolute values of $k_{1}$ and $k_{2}$. In other words, if $k_{1}$ and $k_{2}$ become $c k_{1}$ and $c k_{2}$ respectively, the frequency ratio, $f_{2} / f_{1}$, remains unchanged.

The qualitative understanding of the relationships between the natural frequency and stiffness can effectively simplify the identification process. Instead of identifying the two unknown stiffnesses simultaneously, the ratio of the two stiffnesses is first to be identified to match the target frequency ratio (Table 1) as closely as possible; then the two stiffness values are multiplied by the same scalar to match the measured fundamental natural frequency in Table 1.

This identification strategy can be easily realized in the finite element analysis which requires an input of the two stiffnesses. A value of $k_{1}$ is given and fixed, and different values of $k_{2}$ are provided based on the ratio of $k_{2} / k_{1}$ varying from 0.01 to 2.0 with an increment of 0.001. A do-loop is used to generate the values of $k_{2}$. Thus, a series of values of $f_{1}$ and $f_{2}$ are calculated for the four Models. The relationships between $f_{2} / f_{1}$ and $k_{2} / k_{1}$ can be presented graphically as shown in Fig. 4 where the target frequency ratio of 2.29 and the minimum frequency ratio are also indicated. The stiffness ratio corresponding to the minimum natural frequency

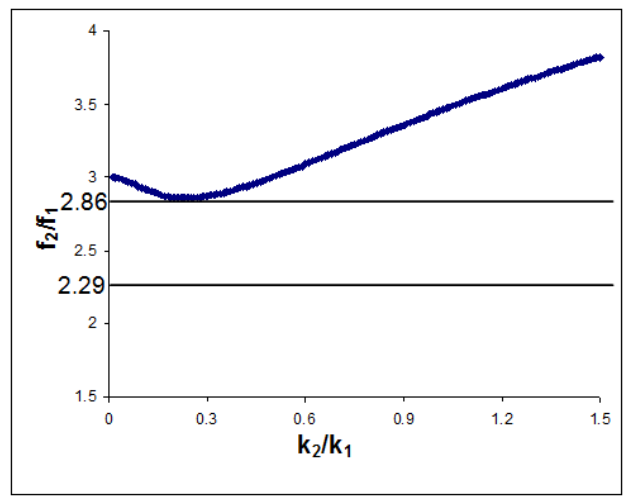

(a) Model 1

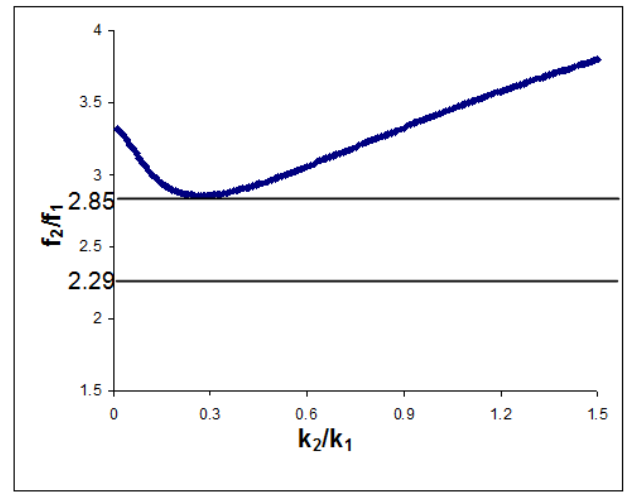

(b) Model 2

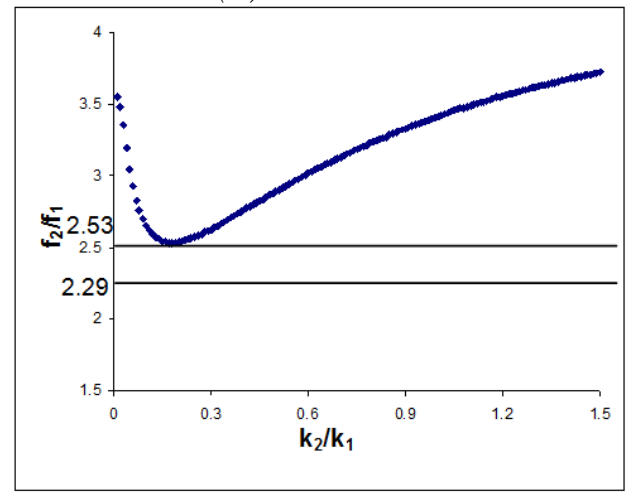

(c) Model 3

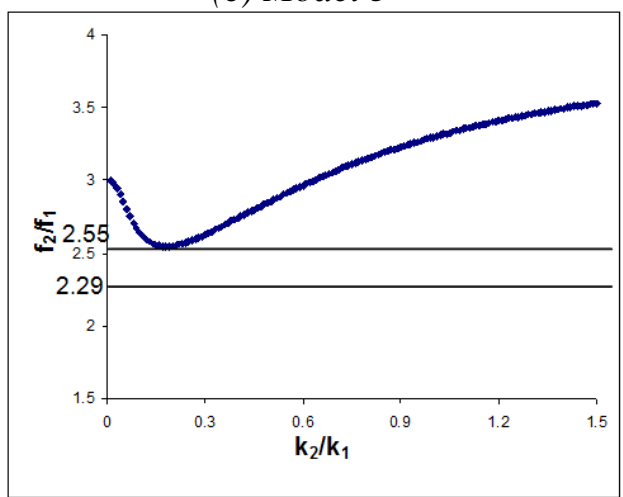

(d) Model 4

Figure 4: Relationships between $k_{2} / k_{1}$ and $f_{2} / f_{1}$ for the four models. 
ratio (Fig. 4) is selected as the solution for each model because the minimum natural frequency ratio is the closest among all values to the target ratio.

If the ratio of the measured natural frequency (5.88 $\mathrm{Hz}$ ) to the calculated fundamental natural frequency corresponding to the minimum frequency ratio is $\mathrm{c}$, the first two calculated natural frequencies are timed by $c$ and the two stiffness values corresponding to the minimum frequency ratio are multiplied by the same factor of $c^{2}$.

Table 3 summarizes the two identified stiffness values and the corresponding first two natural frequencies of the four models together with a comparison between the minimum and targeted frequency ratios.

\subsection{Comparison of the models}

The results given in Table 3 provide an improved understanding of the four continuous body models. It can be observed from Table 3 that:

- The difference between Model 1 and Model 2 is the mass distribution. The results of the two models indicate that the effect of mass distribution on the ratio of the two natural frequencies is not significant (2.86 for Model 1 and 2.85 for Model $2)$. This observation is confirmed by comparing the same for Models 3 and 4 (2.53 for Model 3 and 2.55 for Model 4)

- The differences between Model 2 and Model 3 are the position of the lower torso to the lower or the upper part of the body and the stiffness distribution. As the effect of mass distribution is insignificant, the change of the frequency ratio from 2.85 to 2.53 is mainly due to the change of the distribution of the stiffness and Model 3 appears better than Model 2.

- The frequency ratio for Model 3 is the smallest among the four Models and is closest to the ratio of the measured natural frequencies. Model 3 has the same fundamental natural frequency as the measured one, while its second natural frequency is $10 \%$ larger than the measurement.

- As only two values of stiffness can be used in the model this limits the accuracy of the model. Models 3 and 4 are better than Models 1 and 2 to represent a standing body as a continuous model. Model 3 is slightly better than Model 4 in terms of accuracy but Model 4 has much simpler mass distribution than Model 3.
Table 3: Stiffnesses and natural frequencies of a standing body

\begin{tabular}{|c|c|c|c|c|c|}
\hline & 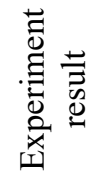 & $\begin{array}{l}\overline{\overline{0}} \\
\overline{0}\end{array}$ & $\frac{N}{\frac{\pi}{0}}$ & $\frac{m}{\frac{m}{0}}$ & $\begin{array}{l}\frac{1}{0} \\
\frac{8}{0} \\
\Sigma\end{array}$ \\
\hline$k_{1}(\mathrm{kN} / \mathrm{m})$ & - & 160.6 & 142.9 & 134.9 & 130.5 \\
\hline$k_{2}(\mathrm{kN} / \mathrm{m})$ & - & 35.33 & 37.15 & 24.01 & 23.36 \\
\hline$f_{\mathrm{H} 1}(\mathrm{~Hz})$ & 5.88 & 5.88 & 5.88 & 5.88 & 5.88 \\
\hline$f_{\mathrm{H} 2}(\mathrm{~Hz})$ & 13.5 & 16.81 & 16.79 & 14.89 & 14.97 \\
\hline$\delta_{i}=\frac{f_{H 2}}{f_{H 1}}$ & 2.29 & 2.86 & 2.85 & 2.53 & 2.55 \\
\hline $\begin{array}{c}\text { Ratio } \\
\delta_{i} / \delta_{0} \\
\left(\delta_{0}=2.29\right)\end{array}$ & - & $125 \%$ & $124 \%$ & $110 \%$ & $111 \%$ \\
\hline
\end{tabular}

\section{Vertical Dynamic Characteristics of a Standing Body}

As Model 3 appears the best of the four, it is used to examine the vertical dynamic characteristics of the standing body.

\subsection{Mode shapes}

The eigenvalue analysis of Model 3 is conducted and the shapes of the first four modes are given in Fig. 5 together with the natural frequencies. It can be observed from Fig. 5 that:

- The first mode of vibration of the standing body is dominated by the upper part (head neck, upper torso and central torso) of the body.

- The fundamental mode shows that all parts of the human body vibrate in the same direction and the head has the maximum movement while the feet the least.

- The second mode shows that the upper torso and the head move in the opposite direction to the other segments. The lower torso has the largest movement while the bottom of the upper torso has little movement.

The third mode shows that the lower parts (the feet, lower legs, upper legs and the lower torso) move in the same direction as the upper torso and the head, while the central torso moves opposite to that of the other segments. 


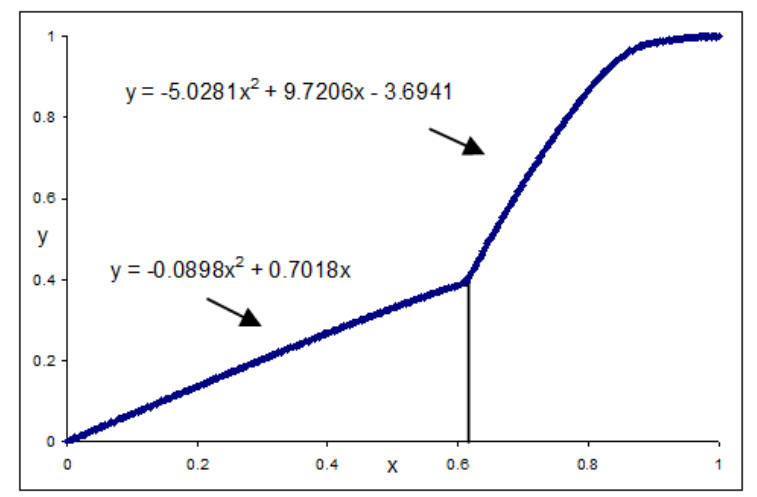

a) The First Mode $(5.88 \mathrm{~Hz})$

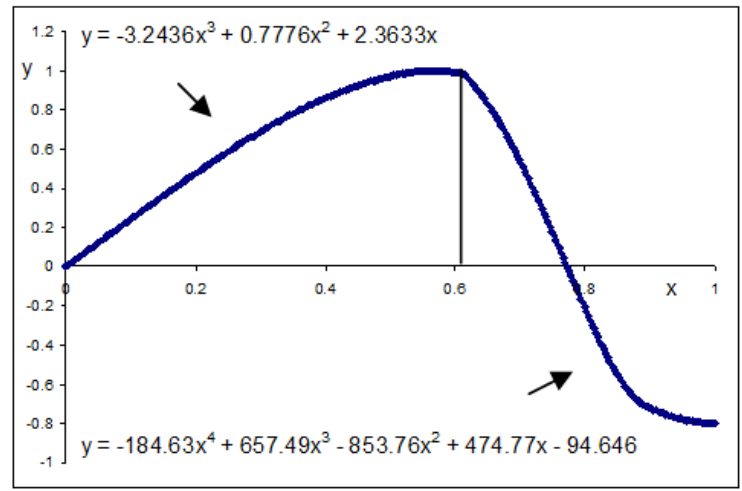

b) The Second Mode $(14.89 \mathrm{~Hz})$

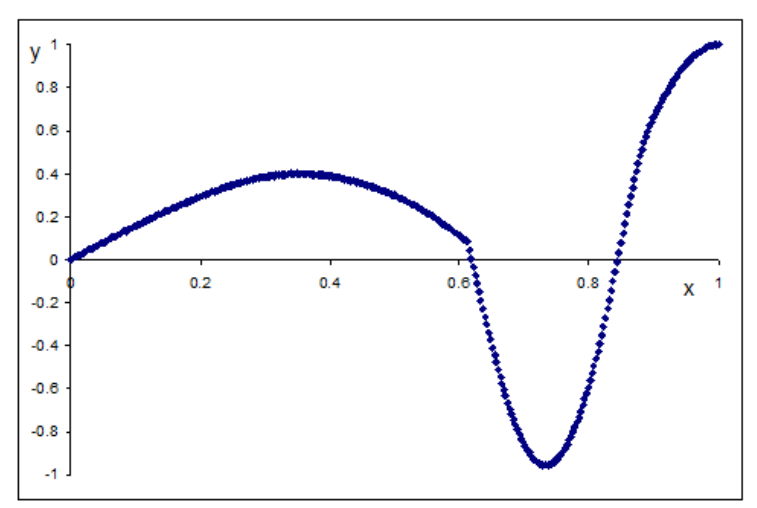

c) The Third Mode $(28.51 \mathrm{~Hz})$

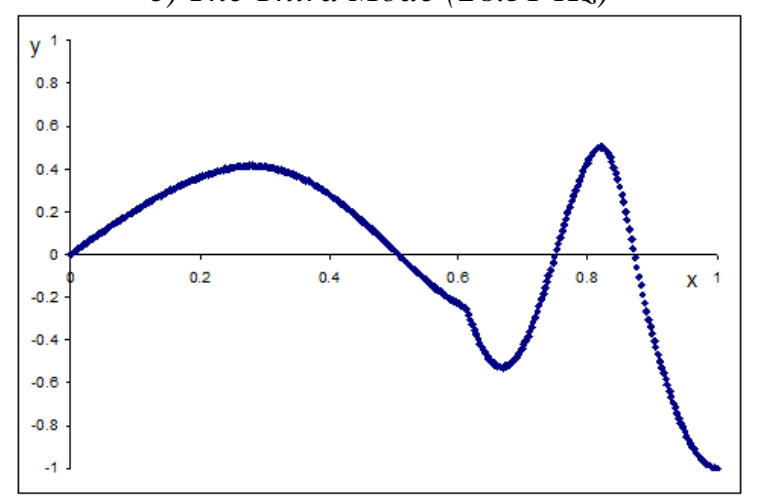

d) The Fourth Mode $(39.87 \mathrm{~Hz})$

Figure 5: The Mode shapes of Model 3.

\subsection{Modal properties}

The modal properties of Model 3 can be calculated from the finite element analysis. The total mass can be determined using the following formula:

$$
M_{\mathrm{H} 0}=\sum_{\mathrm{j}=1}^{n} M_{\mathrm{j}}
$$

Where $M_{\mathrm{i}}$ is the $\mathrm{i}^{\text {th }}$ element and is the total number of elements of the model. The participating factor and modal mass of the model are according to the definitions:

$$
\begin{gathered}
M_{H i}=\sum_{j=1}^{n} M_{j} \phi_{i, j} \quad M_{H i i}=\sum_{\mathrm{i}=\mathrm{j}}^{\mathrm{n}} M_{\mathrm{j}} \phi_{\mathrm{i}, \mathrm{j}}^{2} \\
\mathrm{i}=1,2
\end{gathered}
$$

where $M_{j}$ is the mass of the $\mathrm{j}^{\text {th }}$ element and $\phi_{\mathrm{i}, \mathrm{j}}$ is the movement at the $\mathrm{j}^{\text {th }}$ node in the $\mathrm{i}^{\text {th }}$ mode. The Model stiffness of the model can be determined using the following formula:

$$
\begin{gathered}
K_{\mathrm{Hi}}=\sum_{\mathrm{j}=1}^{\mathrm{n}+1} k_{\mathrm{i}}\left(\frac{\phi_{\mathrm{i}, \mathrm{j}+1}-\phi_{\mathrm{i}, \mathrm{j}}}{\Delta x}\right)^{2} \Delta x \\
=\sum_{\mathrm{j}=1}^{\mathrm{n}+1} k_{\mathrm{i}} \frac{\left(\phi_{\mathrm{i}, \mathrm{j}+1}-\phi_{\mathrm{i}, \mathrm{j}}\right)^{2}}{\Delta x} \\
i=1,2
\end{gathered}
$$

where $\Delta x$ is the length of the element. The modal mass and stiffness for the first two modes of Model 3 are given in Table 4. The last column in the table shows the ratio of the mass properties to the total mass. These modal parameters can be used for the human-structure interaction model shown in Fig. 1b.

\section{Conclusion}

The continuous standing body models in the vertical vibration are developed using the two available natural frequencies of biomechanics model and the anthropomorphic model. Four similar models are considered and assessed in the paper. The most appropriate vertical stiffnesses of the models are identified based on the best matching with the measured natural frequencies. The study shows that the effect of the stiffness distribution is more 
Table 4: Modal mass and stiffness of a standing body

\begin{tabular}{|c|c|c|c|}
\hline \multirow{2}{*}{ Model number } & \multicolumn{2}{|c|}{ Model 3 } \\
\cline { 2 - 4 } & Results & $\begin{array}{c}\text { Ratio } \\
\text { to the } \\
\text { total } \\
\text { mass }\end{array}$ \\
\hline \multicolumn{1}{|c|}{ Total mass $M_{\mathrm{H} 0}(\mathrm{~kg})$} & 74.9 & \\
\hline \multirow{4}{*}{$\begin{array}{c}\text { First } \\
\text { Mode }\end{array}$} & $\begin{array}{c}\text { Mode factor } M_{\mathrm{H} 1} \\
(\mathrm{~kg})\end{array}$ & 40.00 & $53.4 \%$ \\
\cline { 2 - 4 } & $\begin{array}{c}\text { Modal mass } \\
M_{\mathrm{H} 11}(\mathrm{~kg})\end{array}$ & 29.34 & $38.2 \%$ \\
\cline { 2 - 4 } & $\begin{array}{c}\text { Modal stiffness } \\
K_{\mathrm{H} 1}(\mathrm{~N} / \mathrm{m})\end{array}$ & 39844 & \\
\hline \multirow{4}{*}{$\begin{array}{c}\text { Second } \\
\text { Mode } \\
M_{\mathrm{H} 2}(\mathrm{~kg})\end{array}$} & $\begin{array}{c}\text { Modal mass } \\
M_{\mathrm{H} 22}(\mathrm{~kg})\end{array}$ & 36.67 & $49.0 \%$ \\
\cline { 2 - 4 } & $\begin{array}{c}\text { Modal stiffness } \\
K_{\mathrm{H} 2}(\mathrm{~N} / \mathrm{m})\end{array}$ & 320064 & \\
\hline
\end{tabular}

significant than that of the mass distribution along the height of the body on the accuracy of the models. The selected model (Model 3 or Model 4) has the same fundamental natural frequency as the measured one but the second natural frequency is about $10 \%$ larger than the measurement.

The continuous model is able to show the shapes of vibration modes throughout the height of the standing body. The fundamental mode shows that the upper part of the body has much more significant movement than the lower part of the body, while the second mode indicates that the low torso has the largest movement but the upper torso moves insignificantly.

The modal properties for the first two modes are also provided based on the continuous model. These parameters can be used for the human-structure interaction model. However, the parameters of the interaction model can be determined more directly through curve fitting to the actual measurements, which would give more accurate representation.

\section{Acknowledgments}

This work was financially supported by the National Natural Science Foundation of China (Grant no. 51508133 and 51508132), which is gratefully acknowledged.

\section{References}

[1] Griffin M J (1990) Handbook of Human Vibration. Academic Press, London, UK.

[2] Matsumoto Y and Griffin M J (2000) Comparison of biodynamic responses in standing and seated human bodies, Journal of Sound and Vibration, 238(4), 691-704.

[3] Wei L and Griffin M J (1998) Mathematical models for the apparent mass of the seated human body exposed to vertical vibration. Journal of Sound and Vibration, 212(5), 855-874.

[4] Matsumoto Y and Griffin M J (2003) Mathematical Models for the apparent masses of standing subjects exposed to vertical wholebody vibration. Journal of Sound and Vibration, 260, 431-451.

[5] Ji, T., Zhou, D., and Zhang, Q. (2013). Models of a standing human body in vertical vibration. Proceedings of the Institution of Civil Engineers: Structures and Buildings, 166(7), 367-378

[6] Ji, T., Wang, D., Zhang, Q., \& Duarte, E. (2014). Presence of Resonance Frequencies in a Heavily Damped Two-Degree-of-Freedom System Journal of Engineering Mechanics, 140(2), 406-415.

[7] Nigam S P and Malik M (1987) A study on a vibratory Model of a human body. Journal of Biomechanical Engineering, 109, 148-153.

[8] Ji T (1995) A continuous Model for the vertical vibration of the human body in a standing position. UK Informal Group Meeting on Human Response to Vibration, Silsoe, UK, September 1995.

[9] Zhou, D., Ji, T., \& Liu, W. (2012). Dynamic characteristics of a standing human on $a$ SDOF structure. Advances in Vibration Engineering, 11(1), 85-98.

[10] Zhou, D., \& Ji, T. (2012). Free vibration of rectangular plates with attached discrete masses. Shock and Vibration: shock and vibration control - crashworthiness - structural dynamics - impact engineering - sound, 19(1), 101-118.

[11] Bartz J. A. and Gianotti C. R. (1975) Computer program to generate dimensional and inertial properties of the human body. Journal of Engineering for Industry, 75, 49-57. 